\title{
Investigation of Liquid Crystal Ripple Using Ericksen-Leslie Theory for Displays Subject to Tactile Force
}

\author{
Y. J. Lee, ${ }^{1}$ T. S. Liu, ${ }^{1}$ Mao-Hsing Lin, ${ }^{2}$ and Kun-Feng Huang ${ }^{2}$ \\ ${ }^{1}$ Department of Mechanical Engineering, National Chiao Tung University, Hsinchu 30010, Taiwan \\ ${ }^{2}$ Innolux Corporation, Tainan 74147, Taiwan \\ Correspondence should be addressed to T. S. Liu; tsliu@mail.nctu.edu.tw
}

Received 25 June 2013; Accepted 7 December 2013

Academic Editor: Farzad Khani

Copyright (c) 2013 Y. J. Lee et al. This is an open access article distributed under the Creative Commons Attribution License, which permits unrestricted use, distribution, and reproduction in any medium, provided the original work is properly cited.

\begin{abstract}
Liquid crystal display panels subjected to tactile force will show ripple propagation on screens. Tactile forces change tilt angles of liquid crystal molecules and alter optical transmission so as to generate ripple on screens. Based on the Ericksen-Leslie theory, this study investigates ripple propagation by dealing with tilt angles of liquid crystal molecules. Tactile force effects are taken into account to derive the molecule equation of motion for liquid crystals. Analytical results show that viscosity, tactile force, the thickness of cell gap, and Leslie viscosity coefficient lead to tilt angle variation. Tilt angle variations of PAA liquid crystal molecules are sensitive to tactile force magnitudes, while those of 5CB and MBBA with larger viscosity are not. Analytical derivation is validated by numerical results.
\end{abstract}

\section{Introduction}

Many physical phenomena exhibited by the nematic phase liquid crystals (LC), such as unusual flow properties or the LC response to electric and magnetic fields, can be studied by treating LC as a continuous medium. Ericksen and Leslie [1-3] formulated general conservation laws and constitutive equations describing the dynamic behavior. Other continuum theories have been proposed, but it turns out that the Ericksen-Leslie theory is the one that is most widely used in discussing the nematic state. Based on the continuum theory of Ericksen-Leslie, this study constructs a theoretical model in order to investigate the tilt angle variation of LC subjected to both electric field and tactile force. The continuum theory for the nematic state flow is established in two dynamical equations-conservation laws and constitutive equations. Both equations are coupled with each other due to the properties of flow and the director of liquid crystal molecules.

Brochard et al. [4] investigated transient distortions in a nematic film by increasing or decreasing of the magnetic field. Lei et al. [5] used the Ericksen-Leslie equation to describe soliton propagation in nematic liquid crystals under shear. Leslie [6] presented a concise but clear derivation of continuum equations commonly employed to describe static and dynamic phenomena in nematic liquid crystals. Gleeson et al. [7] proposed a two-dimensional theory in which the excitations are fronts between distinct solutions of the steady-state Ericksen-Leslie equations. Lin and Liu [8] use the Ericksen-Leslie equation to describe the flow of liquid crystal material and prove the global existence of weak solutions. De Andrade Lima and Rey [9] proposed computational modeling of the steady capillary Poiseuille flow of flow-aligning discotic nematic liquid crystals; using the Ericksen-Leslie equations predicts solution multiplicity and multistability. Nie et al. [10] used a cell gap and surface dynamic method to derive analytical expressions of LC response time under finite anchoring energy conditions. Cruz et al. [11] used a finite difference technique, based on a projection method, which is developed for solving the dynamic three-dimensional Ericksen-Leslie equations for liquid crystals subject to a strong magnetic field.

Liquid crystal display panels subjected to tactile force will show ripple propagation on screens. Tactile forces change tilt angles of liquid crystal molecules and alter optical transmission so as to generate ripple on screens. Based on the Ericksen-Leslie theory, this paper aims to develop LC dynamics subject to tactile force. Tactile force effects are taken into account to derive the molecule director equation 


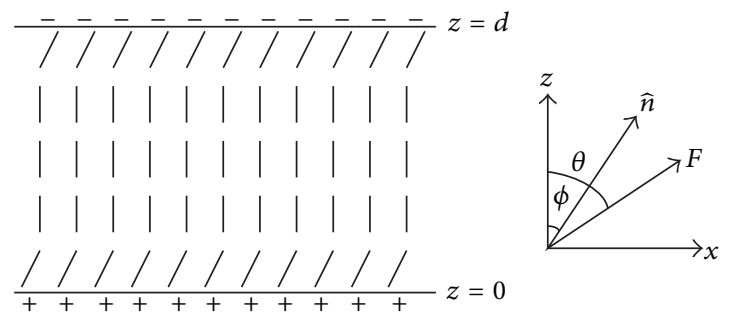

FIGURE 1: Homeotropic alignment LC layer sandwiched between parallel substrates, where $z=0$ and $z=d$ are the bottom and top substrates, respectively.

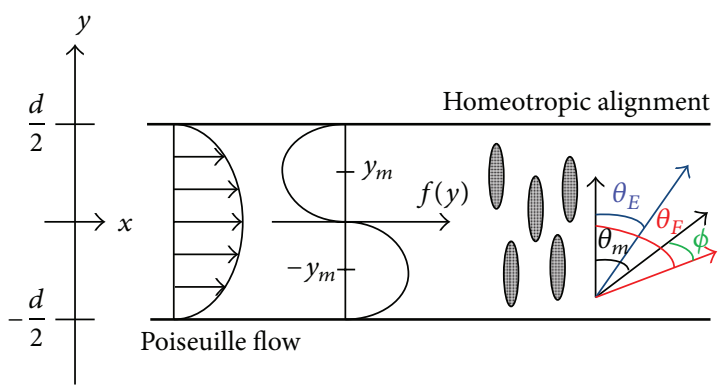

FIGURE 2: Directors of LC molecules with homeotropic alignment are changed by Poiseuille flow.

of motion for liquid crystals. A theoretical model is thus proposed to analyze the tilt angle of nematic liquid crystals subject to tactile forces.

\section{Derivation of Ericksen-Leslie Theory}

2.1. One-Dimensional Model. Figure 1 shows a homeotropic alignment liquid crystal layer sandwiched between parallel substrates, where $z=0$ and $z=d$ stand for the bottom and top substrates, respectively [12]. The thickness between bottom and top substrates is $d$. The $z$-axis is normal to the plane of the substrates, and the electric field $E=8.9 \mathrm{~V}$ is along the $z$-axis. $\theta$ denotes a tilt angle caused by a tactile force and $\phi$ is the tilt angle defined as the angle between the $z$-axis and LC directors. The dynamics of director-axis rotation is described by an Ericksen-Leslie equation [13, 14]:

$$
\begin{aligned}
\frac{\partial}{\partial z}[ & \left.\left(K_{11} \cos ^{2} \theta+K_{33} \sin ^{2} \theta\right) \frac{\partial \theta}{\partial z}\right] \\
& +\left(K_{33}-K_{11}\right) \sin \theta \cos \theta\left(\frac{\partial \theta}{\partial z}\right)^{2} \\
& +\left(\alpha_{2} \sin ^{2} \theta-\alpha_{3} \cos ^{2} \theta\right) \frac{\partial v}{\partial z} \\
& +\varepsilon_{0} \varepsilon_{a} E^{2} \sin \theta \cos \theta=\gamma \frac{\partial \theta}{\partial t}+M \frac{\partial^{2} \theta}{\partial t^{2}},
\end{aligned}
$$

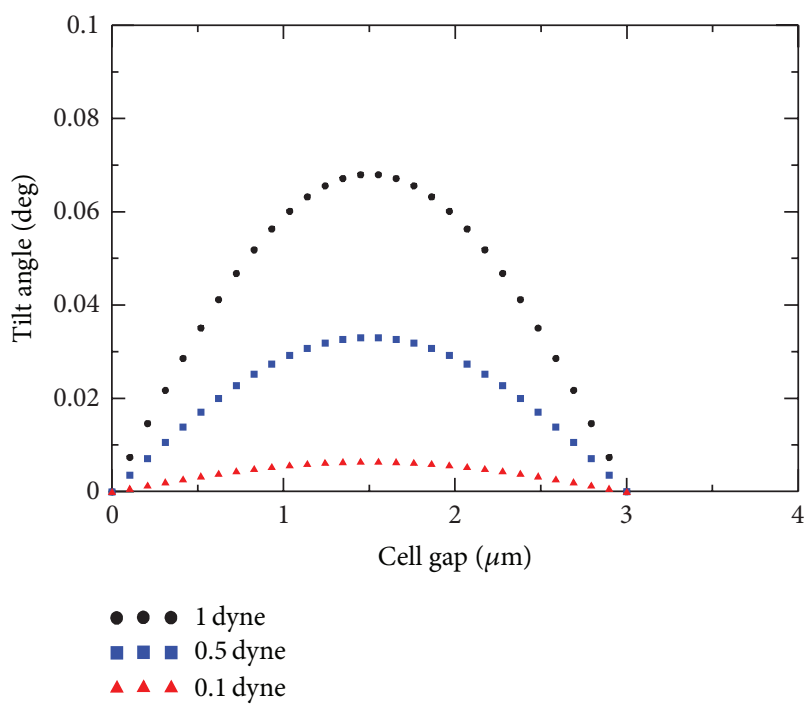

(a)

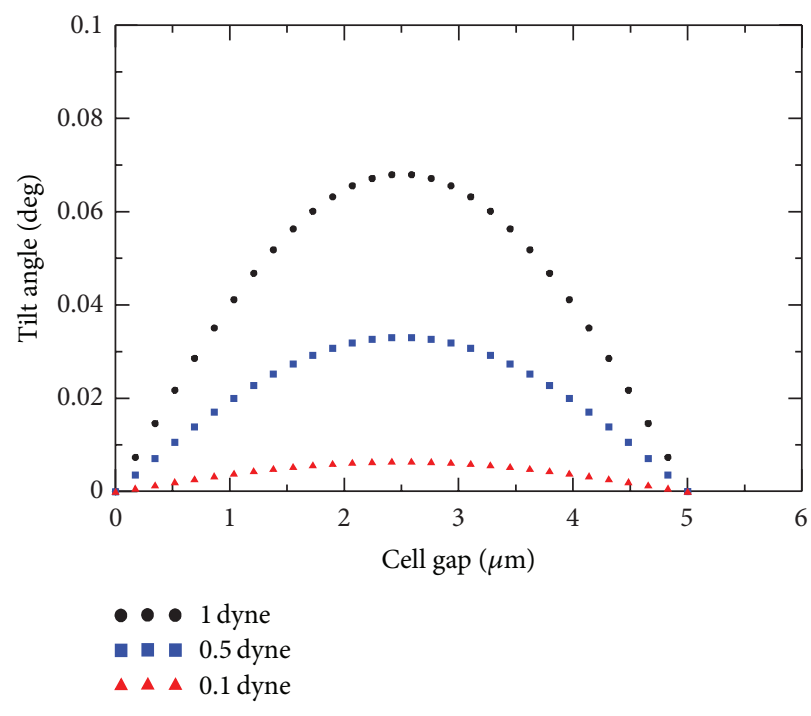

(b)

Figure 3: Comparison of molecule tilt angles between cell gap thicknesses of (a) $3 \mu \mathrm{m}$ and (b) $5 \mu \mathrm{m}$ when a tactile force is applied to LCD screen with 5 CB liquid crystals. 

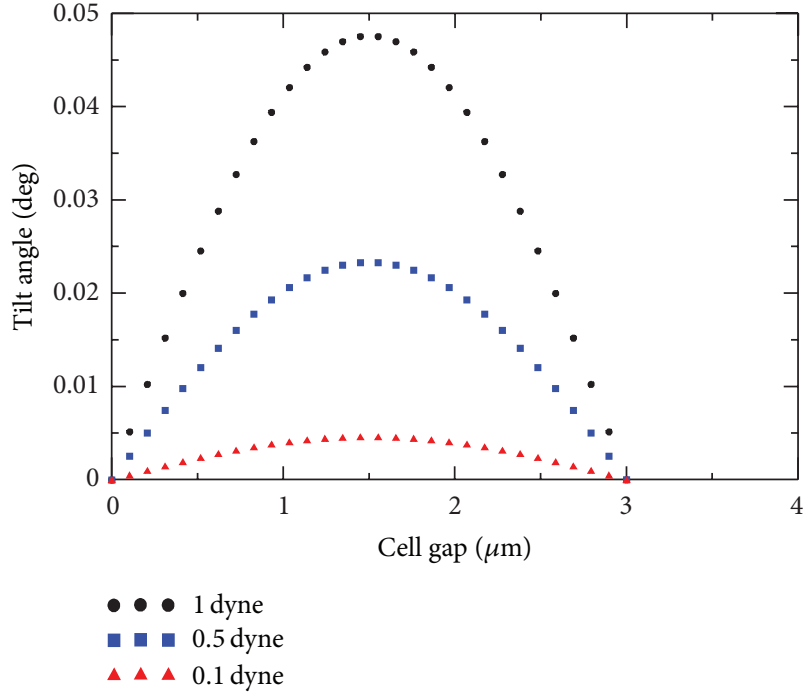

(a)

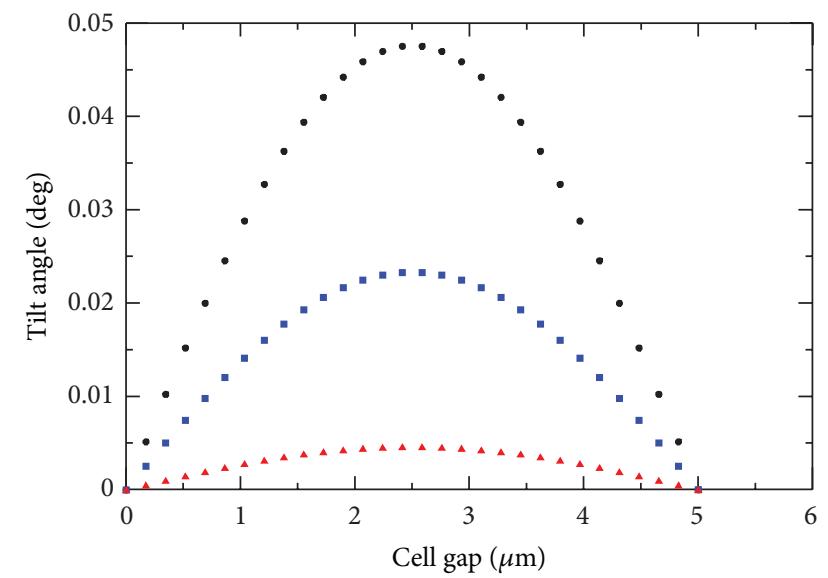

- 1 dyne

- 0.5 dyne

$\Delta \Delta 0.1$ dyne

(b)

Figure 4: Comparison of molecule tilt angles between cell gap thicknesses of (a) $3 \mu \mathrm{m}$ and (b) $5 \mu \mathrm{m}$ when a tactile force is applied to LCD screen with MBBA liquid crystals.

where $K_{11}, K_{22}$, and $K_{33}$ are elastic constants, $\alpha_{2}$ and $\alpha_{3}$ are Leslie viscosities, $v$ is the flow velocity, $\gamma=\alpha_{3}-\alpha_{2}$ is the rotational viscosity, and $M$ is the inertia of LC directors. If backflows, inertial effects, and electric field energy densities are ignored, (1) is reduced to

$$
\gamma \frac{\partial \theta}{\partial t}=K \frac{\partial^{2} \theta}{\partial z^{2}}+F \times \widehat{n},
$$

where $F$ is the tactile force, $K$ is the one-constant approximation with $K=K_{11}=K_{22}=K_{33}$, and $\hat{n}$ is the LC director. Equation (2) is a torque balance equation relating the viscosity, the elasticity, and the torque due to tactile force. A cross product $F \times \widehat{n}$ represents a torque expressed by

$$
F \times \widehat{n}=|F||n| \sin (\theta-\phi),
$$

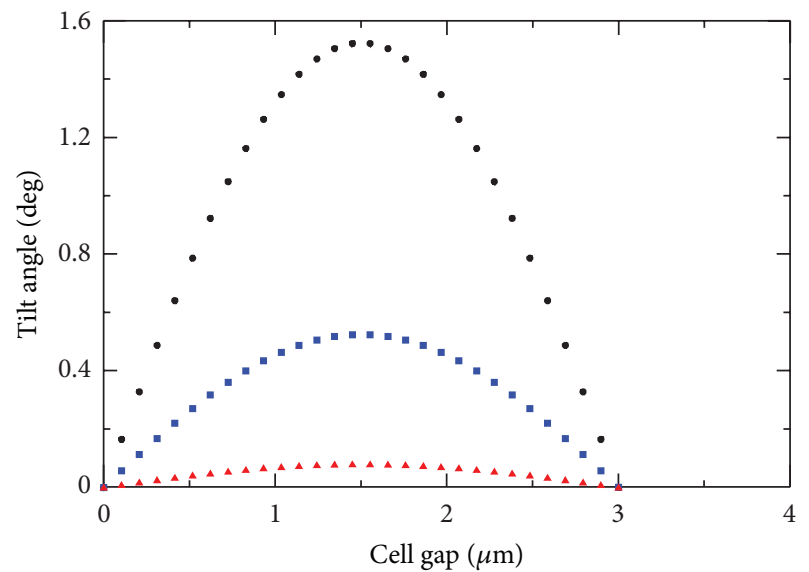

- 1 dyne

- 0.5 dyne

$\triangle \Delta$ A 0.1 dyne

(a)

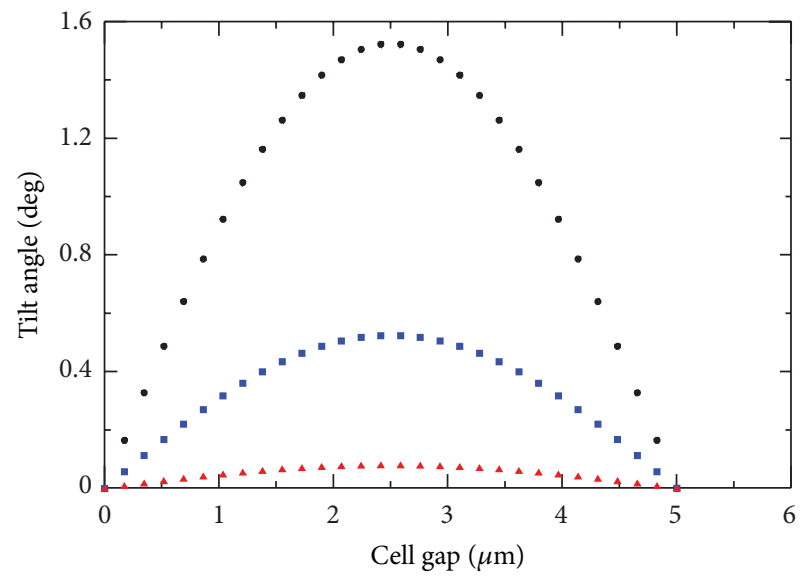

- 1 dyne

- 0.5 dyne

$\Delta \Delta$ - 0.1 dyne

(b)

FIGURE 5: Comparison of molecule tilt angles among cell gap thicknesses of (a) $3 \mu \mathrm{m}$ and (b) $5 \mu \mathrm{m}$ when a tactile force is applied to LCD screen with PAA liquid crystals.

where $|n|=1$. Substituting (3) into (2) gives

$$
\gamma \frac{\partial \theta}{\partial t}=K \frac{\partial^{2} \theta}{\partial z^{2}}+F \sin (\theta-\phi)
$$

Liquid crystal dynamics is described in this study by considering the following two cases.

Case 1. When torque due to tactile force is much larger than elastic torque,

$$
|F \sin (\theta-\phi)| \gg\left|K \frac{\partial^{2} \theta}{\partial z^{2}}\right|
$$



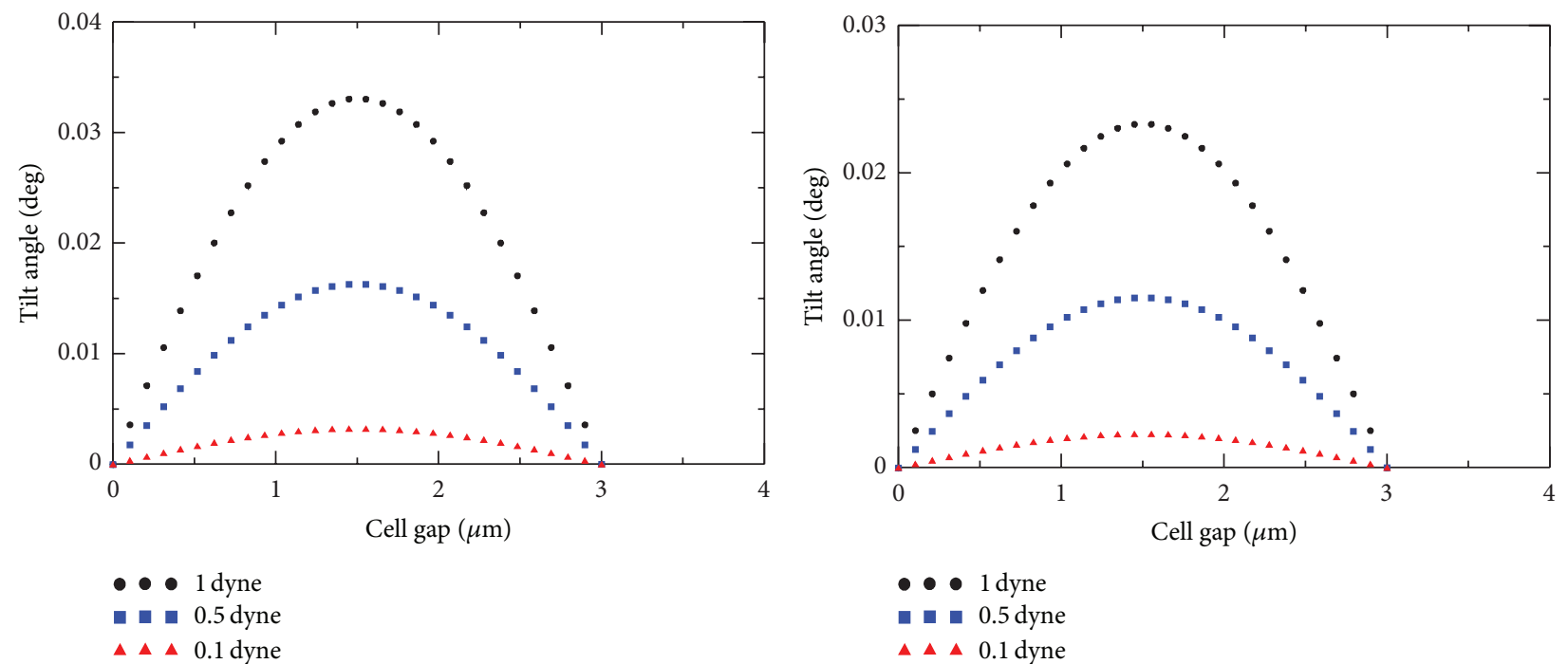

- 1 dyne

0.5 dyne

$\Delta \Delta \quad 0.1$ dyne

(a)

(b)
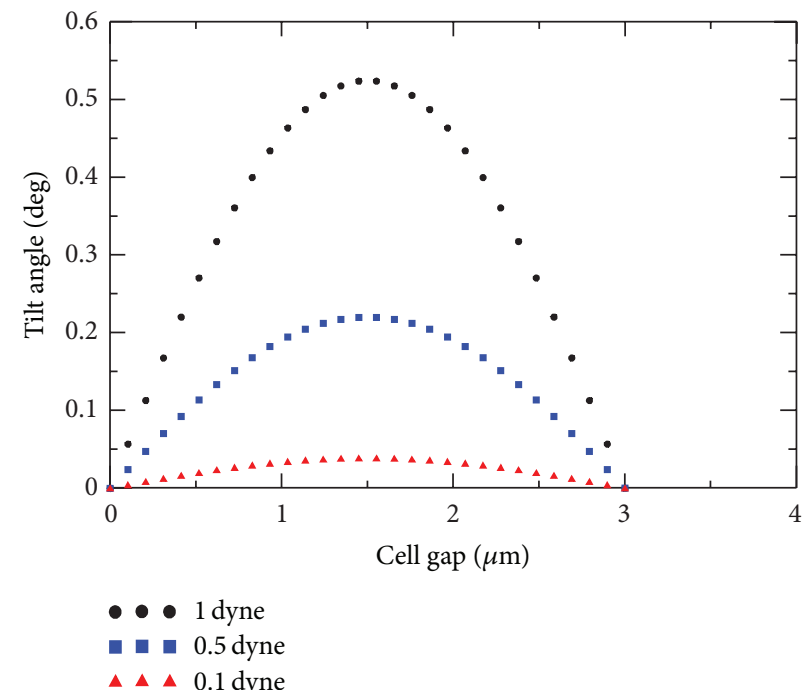

(c)

FIGURE 6: Comparison of molecule tilt angles with different LC materials (a) 5CB, (b) MBBA, and (c) PAA when a tactile force is applied to LCD screen.

However, when the tilt angle is small, $\sin \theta \approx \theta$ and $\cos \theta \approx 1$ and (4) becomes

$$
\gamma \frac{\partial \theta}{\partial t}=F \cdot(\cos \phi) \cdot \theta-F \cdot \sin \phi
$$

Case 2. When torque due to tactile force is much smaller than elastic torque,

$$
|F \sin (\theta-\phi)| \ll\left|K \frac{\partial^{2} \theta}{\partial z^{2}}\right| .
$$

Equation (4) thus becomes

$$
\gamma \frac{\partial \theta}{\partial t}=K \frac{\partial^{2} \theta}{\partial z^{2}} .
$$

Based on separation of variables, assume $\theta(t, z)=$ $\theta(t) \sin (\pi z / d)$, which is in turn substituted into (6) and (8). Case 1 thus leads to an equation for $\theta$ of the form

$$
\dot{\theta}=a+b \theta,
$$

where

$$
\begin{gathered}
a=-\frac{F \sin \phi}{\gamma}, \\
b=\frac{F \cos \phi}{\gamma} .
\end{gathered}
$$

The solution for $\theta(t, z)$ is written as

$$
\theta(t, z)=\tan \phi\left(e^{(F \cos \phi / \gamma) t}\right) \sin \left(\frac{\pi z}{d}\right)
$$




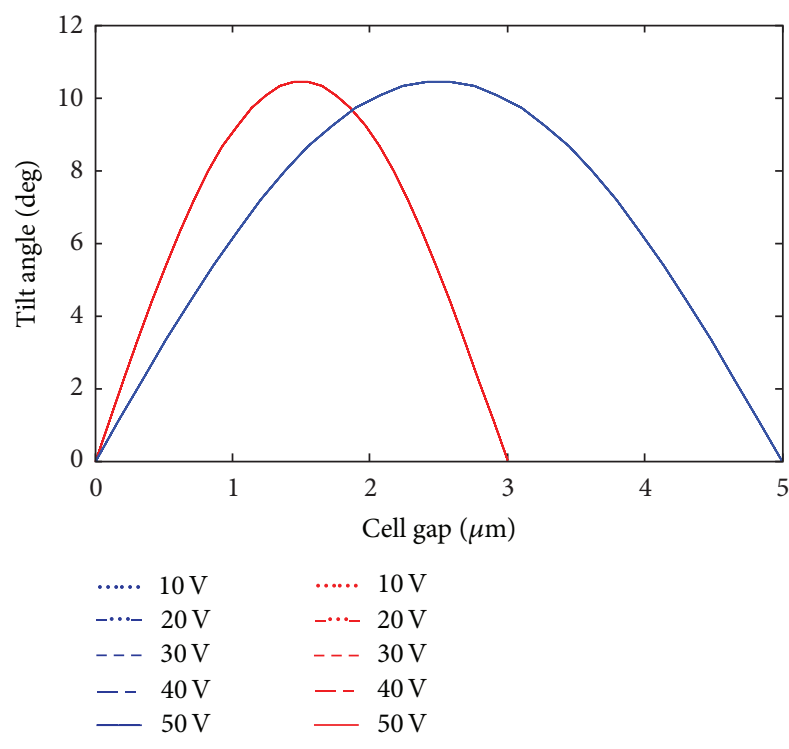

(a)

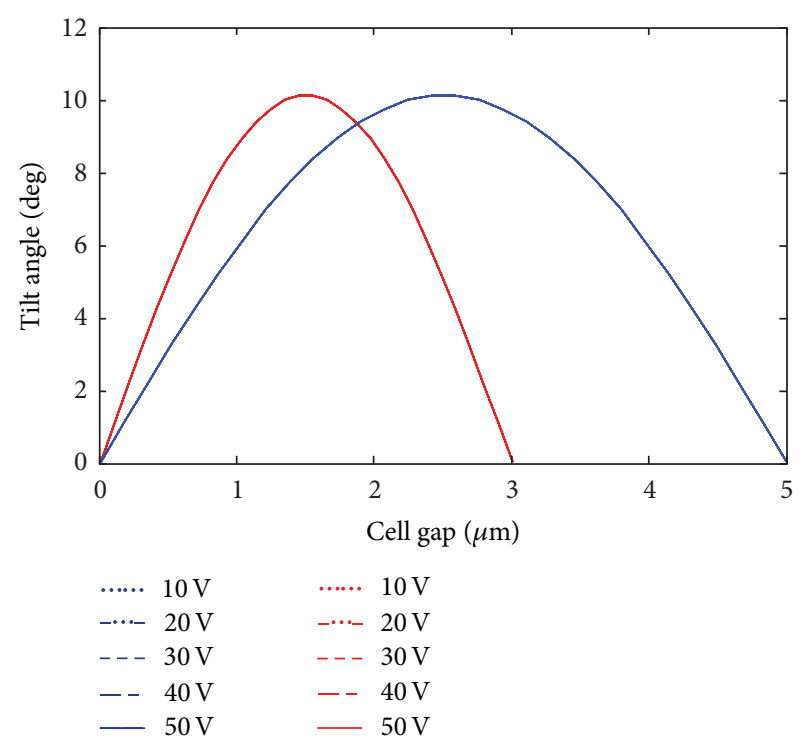

(b)

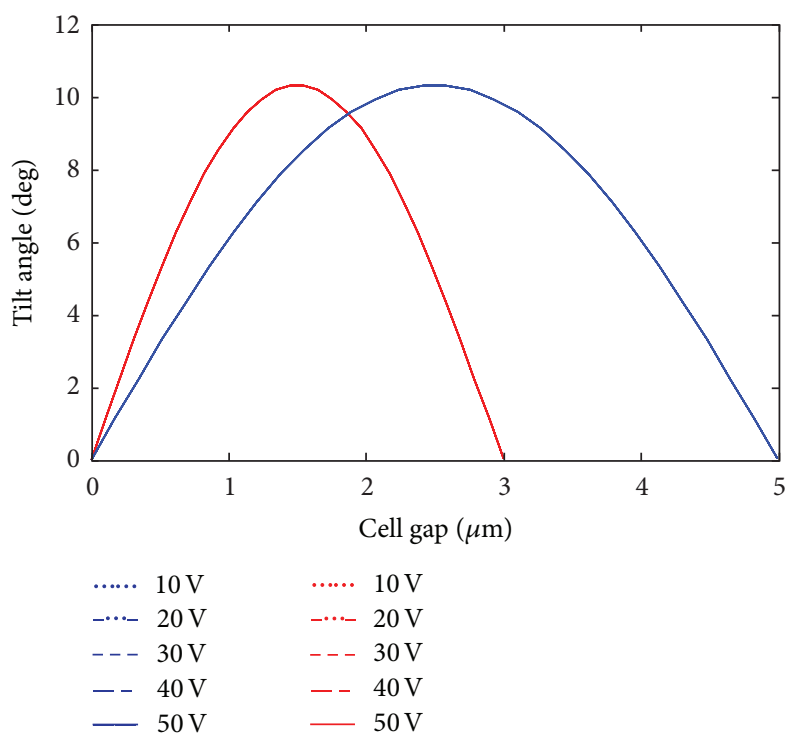

(c)

FIGURE 7: Comparison of molecule tilt angles between cell gap thicknesses of $3 \mu \mathrm{m}$ and $5 \mu \mathrm{m}$ when tactile force is applied to LCD screen with (a) 5CB, (b) MBBA, and (c) PAA liquid crystals under different voltages.

In Case 2, which happens when the tactile force is negligible, the solution for $\theta(t, z)$ in (8) is expressed by

$$
\theta(t, z)=c e^{-\left(K \pi^{2} / \gamma d^{2}\right) t} \sin \left(\frac{\pi z}{d}\right),
$$

where $c$ is a constant. Since the tactile force is ignored in Case 2, only Case 1 is further examined.

2.2. Two-Dimensional Model. The above derivation is based on 1D LC model. Further, this study intends to use (1) to develop an LC tilt model in two dimensions. Assume that the theoretical model satisfies the following four conditions, as depicted in Figure 2.
Condition 1. The molecule ignores the moment of inertia; that is, $M=0$.

Condition 2. Shear rate $s=s(y)=\partial v / \partial y=v /(d / 3)$, where $v$ is the flow velocity and $d$ is the cell thickness.

Condition 3. The LC sample is homeotropic alignment, and we have the boundary conditions

$$
\theta(x, y, t)=\theta\left(x, \pm \frac{d}{2}, t\right)=0 .
$$

Condition 4. The shearing velocity belongs to Poiseuille flow [9]. 


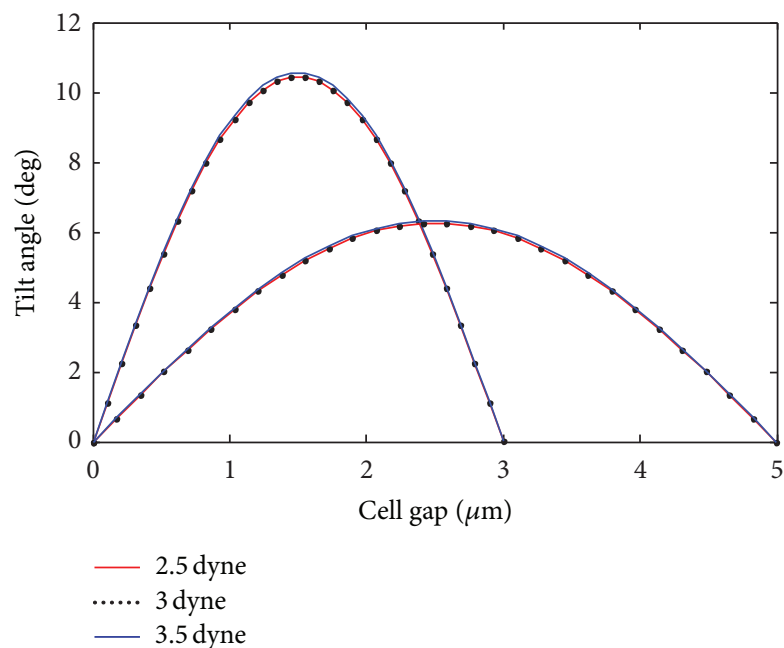

(a)

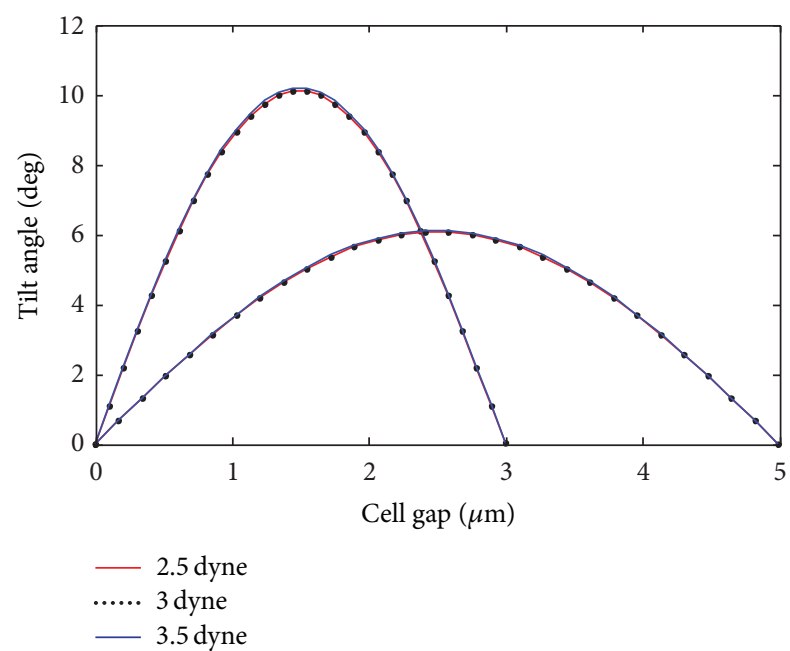

(b)

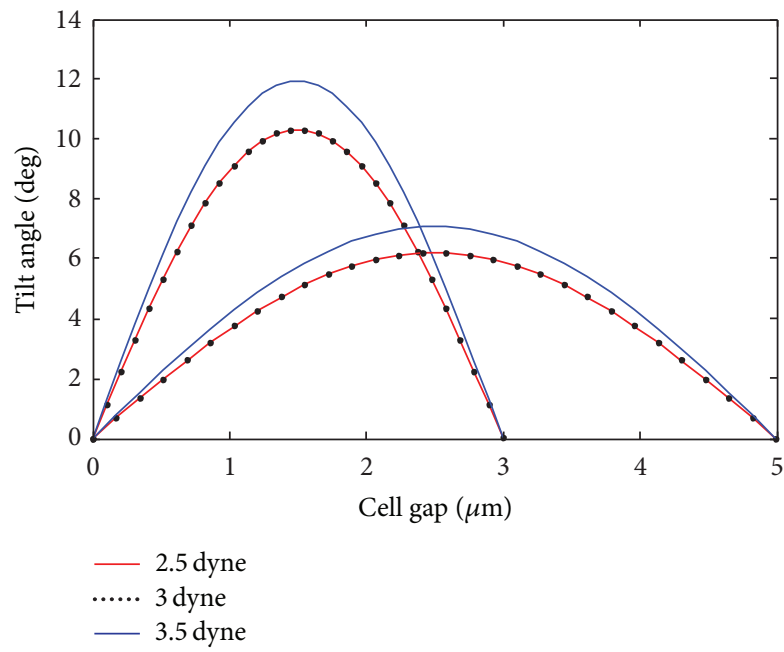

(c)

Figure 8: Comparison of molecule tilt angles between cell gap thicknesses of $3 \mu \mathrm{m}$ and $5 \mu \mathrm{m}$ when tactile force is applied to LCD screen with (a) 5CB, (b) MBBA, and (c) PAA liquid crystals under different tactile forces.

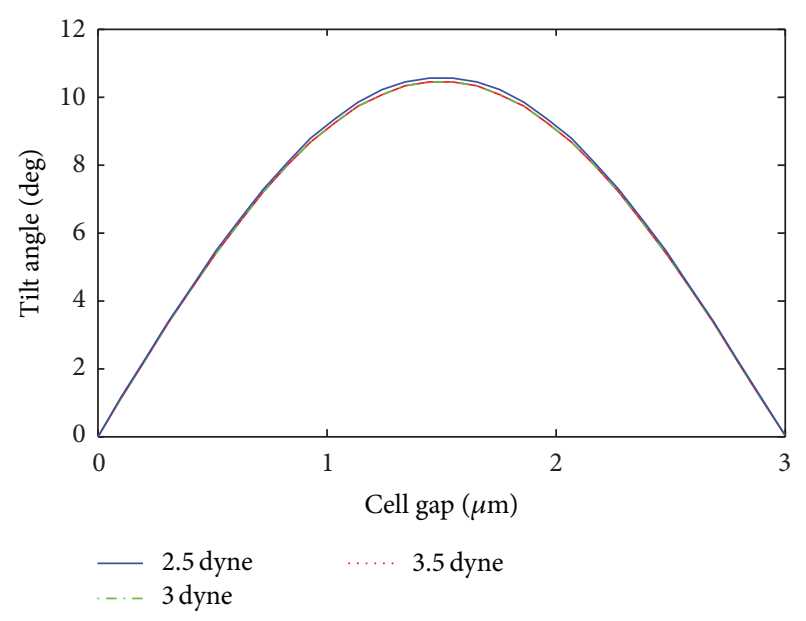

(a)

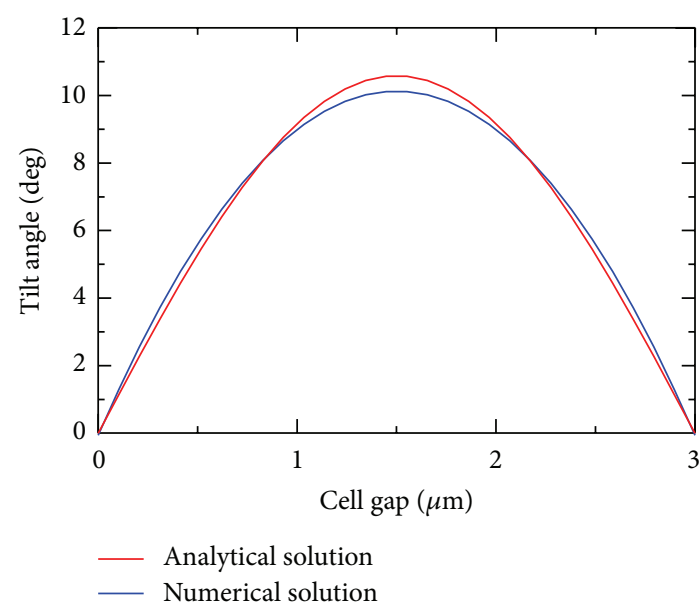

(b)

FIgure 9: (a) Comparison of molecule tilt angles depicted in $3 \mu \mathrm{m}$ cell gap 5CB liquid crystals subject to different forces. (b) Comparison of molecule tilt angles subject to 3.5 dyne between analytical and numerical results.

According to the above assumptions, (1) becomes

$$
\begin{gathered}
K\left(\frac{\partial^{2} \theta}{\partial x^{2}}+\frac{\partial^{2} \theta}{\partial y^{2}}\right)-\gamma \frac{\partial \theta}{\partial t}+\frac{s}{2}\left(\gamma_{1}-\gamma_{2} \cos 2 \theta\right) \\
+\varepsilon_{0} \varepsilon_{a} E^{2} \sin \theta \cos \theta+F \times \widehat{n}=0 .
\end{gathered}
$$

Based on the Ericksen-Leslie theory, (14) represents the tilt model of LC molecule subjected to both electric field and tactile force. In order to use separation of variables, assume $\theta=\theta(x, y, t)$ yields

$$
\theta(x, y, t)=f(y) \theta_{m}(x, t)
$$




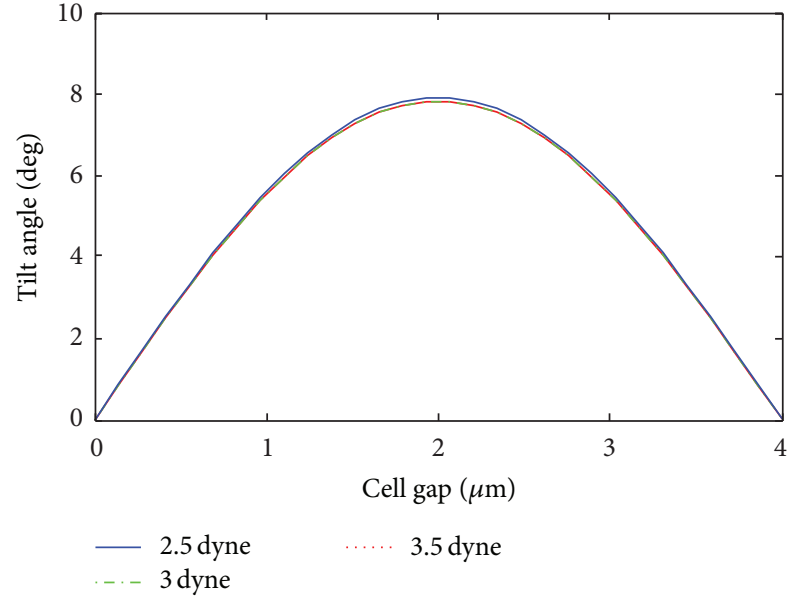

(a)

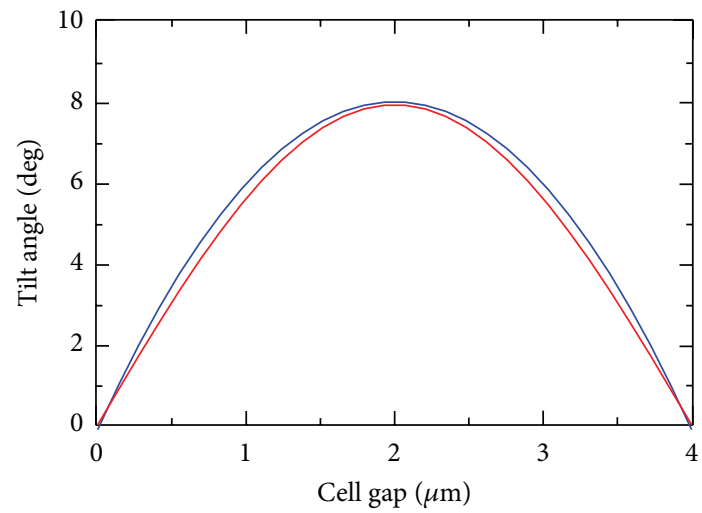

- Analytical solution

Numerical solution

(b)

FIgure 10: (a) Comparison of molecule tilt angles depicted in $4 \mu \mathrm{m}$ cell gap $5 \mathrm{CB}$ liquid crystals subject to different forces. (b) Comparison of molecule tilt angles subject to 3.5 dyne between analytical and numerical results.

where

$$
\begin{gathered}
f(y)=\frac{g(y)}{g\left(-y_{m}\right)} \\
= \begin{cases}1 \text { or }-1, & y=-y_{m} \text { or } y_{m} \\
\frac{g(y)}{g(-0.28 d)}=4.7503 \cdot g(y), & y \neq-y_{m} \text { or } y_{m},\end{cases} \\
g(y)=\frac{2 y}{d}-\sin \left(\frac{y \pi}{d}\right), \\
y_{m}=\left(\frac{d}{\pi}\right) \cos ^{-1}\left(\frac{2}{\pi}\right) \approx 0.28 d,
\end{gathered}
$$

and $y_{m}$ and $-y_{m}$ are boundary conditions of function $g(y)$. In (15), $\theta_{m}$ is the angle of the normal vector between the director of LC molecules with homeotropic alignment. Molecule

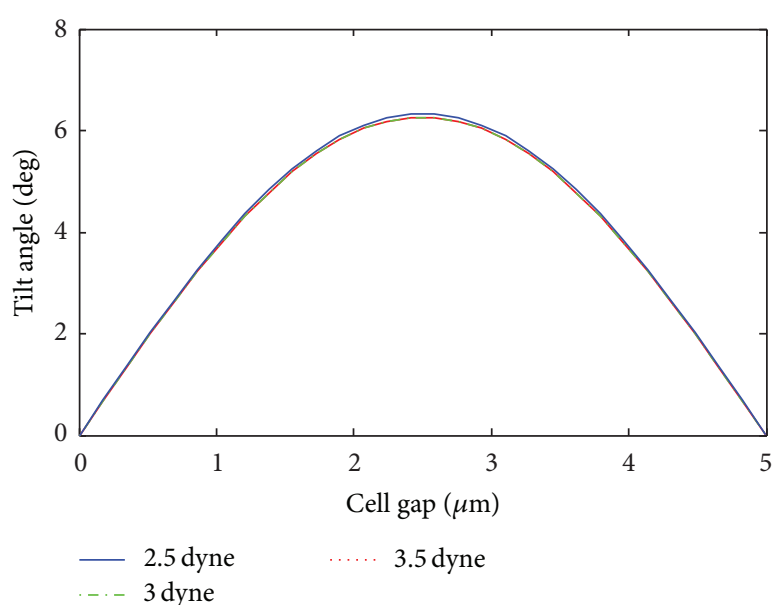

(a)

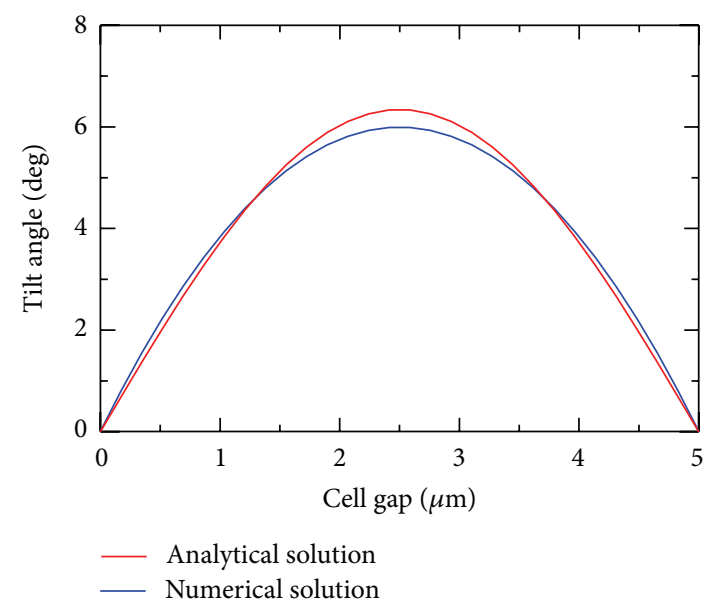

(b)

FIgURE 11: (a) Comparison of molecule tilt angles depicted in $5 \mu \mathrm{m}$ cell gap $5 \mathrm{CB}$ liquid crystals subject to different forces. (b) Comparison of molecule tilt angles subject to 3.5 dyne between analytical and numerical results.

directions are changed by the Poiseuille flow. Values of the angle along the $y$-axis have extreme values at $y_{m}$ and $-y_{m}$. Substituting (15)-(18) into (14) gives

$$
\begin{gathered}
K\left[\frac{\partial^{2}}{\partial x^{2}}\left(f(y) \theta_{m}(x, t)\right)+\frac{\partial^{2}}{\partial y^{2}}\left(f(y) \theta_{m}(x, t)\right)\right] \\
-\gamma \frac{\partial}{\partial t}\left(f(y) \theta_{m}(x, t)\right)+\frac{\partial v}{\partial y}\left(\alpha_{3} \sin ^{2} \theta-\alpha_{2} \cos ^{2} \theta\right) \\
+\varepsilon_{0} \varepsilon_{a} E^{2} \sin \theta \cos \theta+|F||n| \sin (\theta-\phi)=0 .
\end{gathered}
$$

Since $|n|=1$, one has

$$
|F||n| \sin (\theta-\phi)=F \cdot \sin (\theta-\phi) .
$$




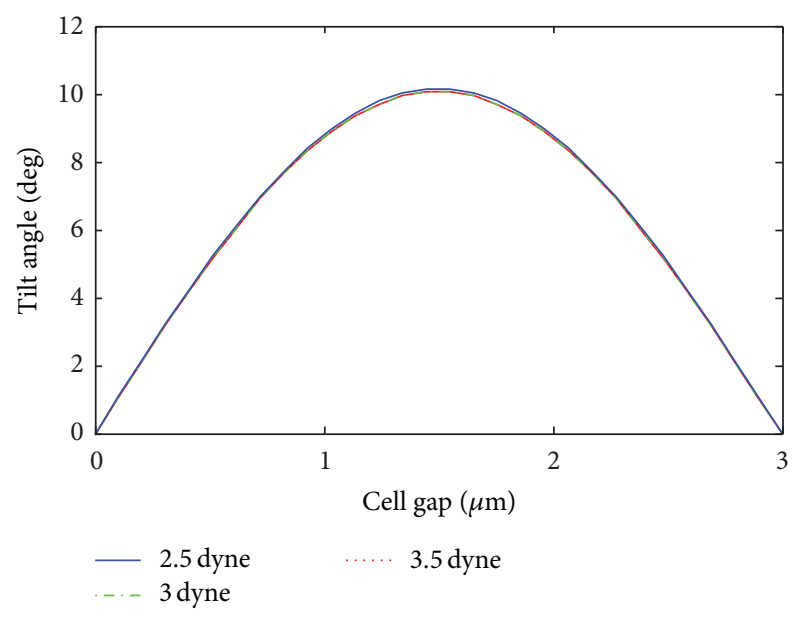

(a)

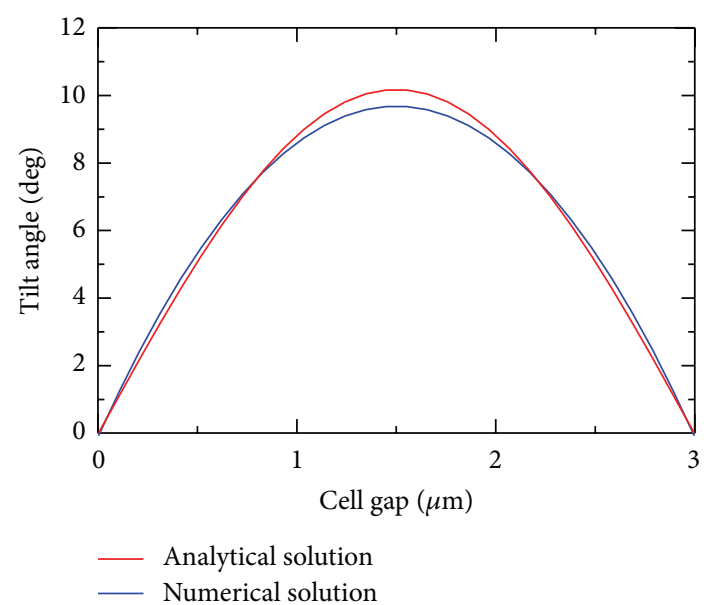

(b)

Figure 12: (a) Comparison of molecule tilt angles depicted in $3 \mu \mathrm{m}$ cell gap MBBA liquid crystals subject to different forces. (b) Comparison of molecule tilt angles subject to 3.5 dyne between analytical and numerical results.

Therefore, (19) becomes

$$
\begin{aligned}
K\left\{\left[f(y) \frac{\partial^{2} \theta_{m}}{\partial x^{2}}+0\right]+\left[\theta_{m} \frac{\partial^{2} f(y)}{\partial y^{2}}+0\right]\right\}-\gamma f(y) \frac{\partial \theta_{m}}{\partial t} \\
\quad+\frac{\partial v}{\partial y}\left[\alpha_{3} \sin ^{2}\left(f(y) \theta_{m}\right)-\alpha_{2} \cos ^{2}\left(f(y) \theta_{m}\right)\right] \\
\quad+\varepsilon_{0} \varepsilon_{a} E^{2} \sin \left(f(y) \theta_{m}\right) \cos \left(f(y) \theta_{m}\right) \\
\quad+F \sin \left(f(y) \theta_{m}-\phi\right)=0 .
\end{aligned}
$$

As depicted in Figure 2, the director makes an angle $\theta_{m}$ with the $y$-axis. Consequently, $\theta_{m}$ represents liquid crystal molecules with homeotropic alignment caused by the Poiseuille flow between director $\widehat{n}$ and normal direction of LC sample. An effective director equation of motion with the essential physics preserved may be obtained by setting $y=-y_{m}$ and $y=y_{m}$ in $(21)$.

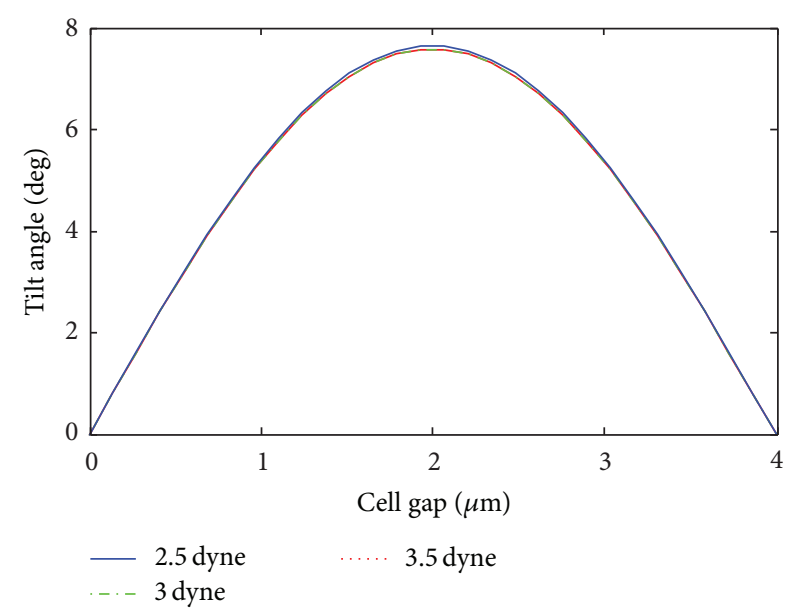

(a)

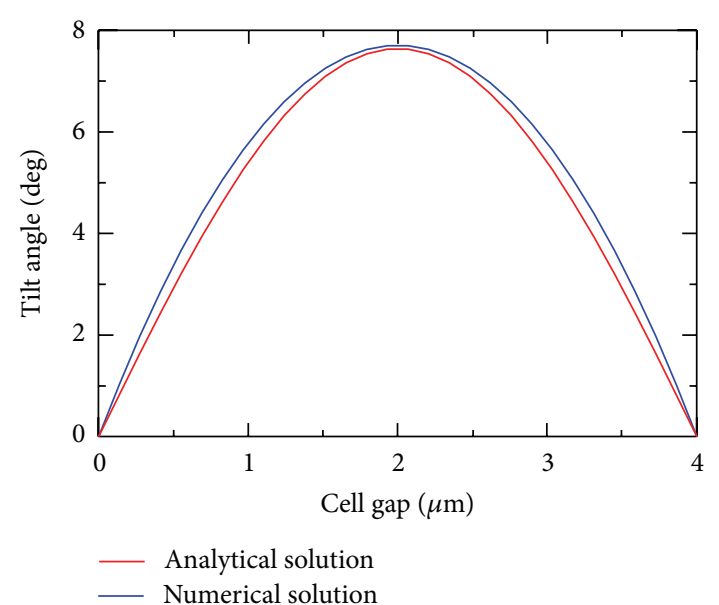

(b)

Figure 13: (a) Comparison of molecule tilt angles depicted in $4 \mu \mathrm{m}$ cell gap MBBA liquid crystals subject to different forces. (b) Comparison of molecule tilt angles subject to 3.5 dyne between analytical and numerical results.

On boundary $y=-y_{m}$ in Figure 2,

$$
\begin{aligned}
& K \frac{\partial^{2} \theta_{m}}{\partial x^{2}}-\gamma \frac{\partial \theta_{m}}{\partial t}+\frac{\partial v}{\partial y}\left(\alpha_{3} \sin ^{2} \theta_{m}-\alpha_{2} \cos ^{2} \theta_{m}\right) \\
& \quad+\varepsilon_{0} \varepsilon_{a} E^{2} \sin \theta_{m} \cos \theta_{m}+F \sin \left(\theta_{m}-\phi\right)=0 .
\end{aligned}
$$

By using trigonometric formulas, one can rewrite (22) as

$$
\begin{aligned}
K \frac{\partial^{2} \theta_{m}}{\partial x^{2}}-\gamma \frac{\partial \theta_{m}}{\partial t}+\frac{\partial v}{\partial y} & \\
\times & {\left[\alpha_{3}\left(\frac{1-\cos 2 \theta_{m}}{2}\right)-\alpha_{2}\left(\frac{1+\cos 2 \theta_{m}}{2}\right)\right] } \\
& +\varepsilon_{0} \varepsilon_{a} E^{2} \theta_{m}+F\left(\sin \theta_{m} \cos \phi-\cos \theta_{m} \sin \phi\right)=0 .
\end{aligned}
$$




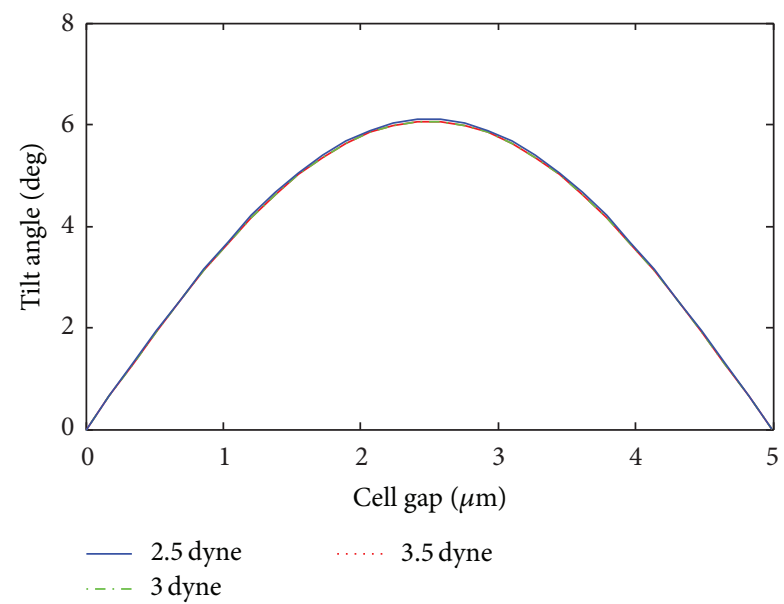

(a)

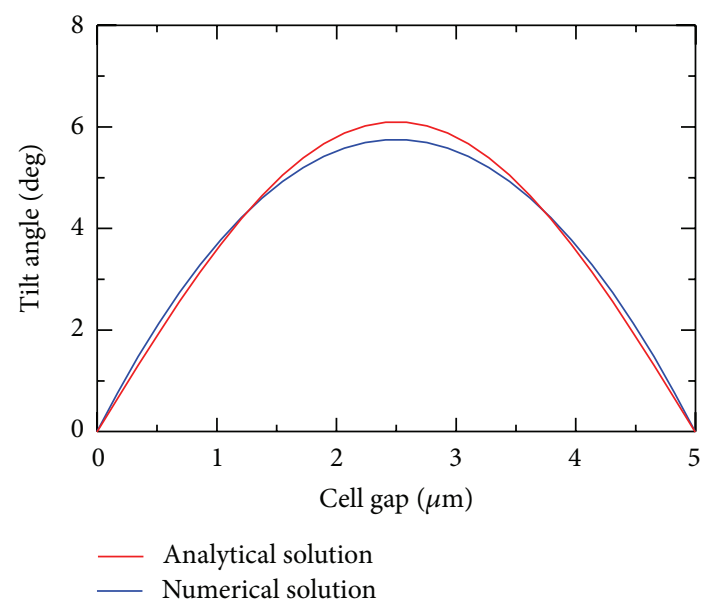

(b)

FIgURE 14: (a) Comparison of molecule tilt angles depicted in $5 \mu \mathrm{m}$ cell gap MBBA liquid crystals subject to different forces. (b) Comparison of molecule tilt angles subject to 3.5 dyne between analytical and numerical results.

Because $\sin \theta_{m} \approx \theta_{m}$ and $\cos \theta_{m} \approx 1$ for small $\theta_{m},(23)$ is rewritten as

$$
\begin{gathered}
K \frac{\partial^{2} \theta_{m}}{\partial x^{2}}-\gamma \frac{\partial \theta_{m}}{\partial t}+\frac{\partial v}{\partial y}\left[\alpha_{3}\left(\frac{1-1}{2}\right)-\alpha_{2}\left(\frac{1+1}{2}\right)\right] \\
+\varepsilon_{0} \varepsilon_{a} E^{2} \theta_{m}+F\left(\theta_{m} \cos \phi-\sin \phi\right)=0 .
\end{gathered}
$$

Thus,

$$
\begin{gathered}
K \frac{\partial^{2} \theta_{m}}{\partial x^{2}}-\gamma \frac{\partial \theta_{m}}{\partial t}-\alpha_{2} \frac{\partial v}{\partial y}+\varepsilon_{0} \varepsilon_{a} E^{2} \theta_{m} \\
+F\left(\theta_{m} \cos \phi-\sin \phi\right)=0 .
\end{gathered}
$$

Hence,

$$
\gamma \frac{\partial \theta_{m}}{\partial t}=K \frac{\partial^{2} \theta_{m}}{\partial x^{2}}-\alpha_{2} \frac{\partial v}{\partial y}+\varepsilon_{0} \varepsilon_{a} E^{2} \theta_{m}+F\left(\theta_{m} \cos \phi-\sin \phi\right) .
$$

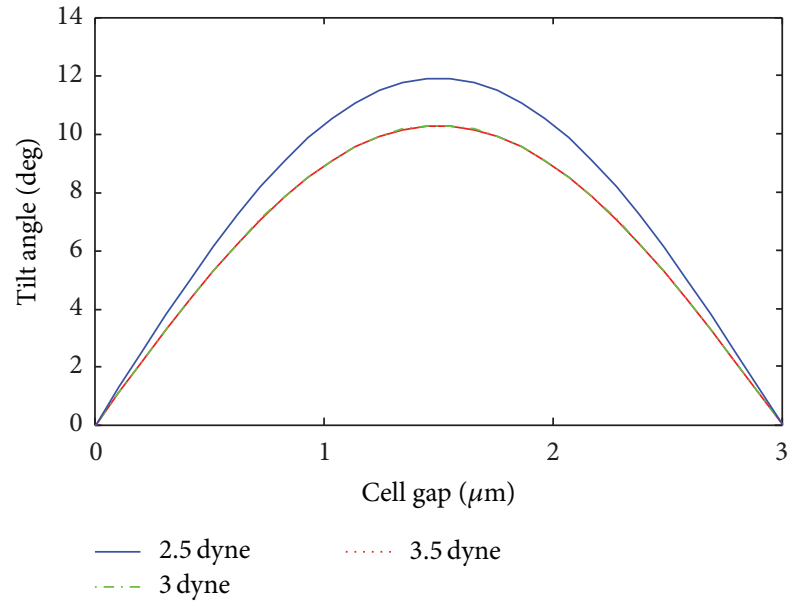

(a)

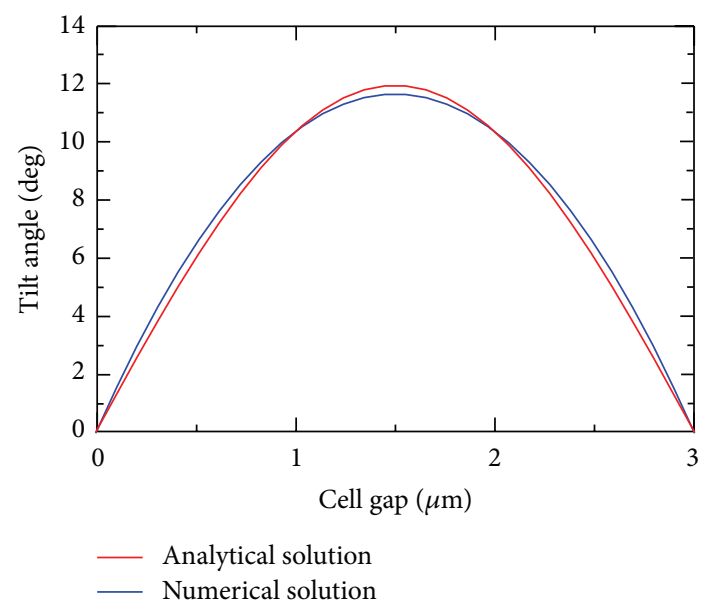

(b)

Figure 15: (a) Comparison of molecule tilt angles depicted in $3 \mu \mathrm{m}$ cell gap PAA liquid crystals subject to different forces. (b) Comparison of molecule tilt angles subject to 3.5 dyne between analytical and numerical results.

Substituting $\theta_{m}=\theta_{m}(t) \sin (\pi x / d)$ for separation of variables into (26) leads to

$$
\begin{aligned}
\gamma \dot{\theta}_{m}= & K \frac{\partial^{2}}{\partial x^{2}}\left[\theta_{m}(t) \sin \left(\frac{\pi x}{d}\right)\right]-\alpha_{2} \frac{\partial v}{\partial y} \\
& +\varepsilon_{0} \varepsilon_{a} E^{2} \theta_{m}+F\left(\theta_{m} \cos \phi-\sin \phi\right) .
\end{aligned}
$$

As a result,

$$
\begin{aligned}
\gamma \dot{\theta}_{m}= & -\frac{K \pi^{2}}{d^{2}} \theta_{m}(x, t)+\left(\varepsilon_{0} \varepsilon_{a} E^{2}+F \cos \phi\right) \theta_{m} \\
& +\left(-\alpha_{2} \frac{\partial v}{\partial y}-F \sin \phi\right) .
\end{aligned}
$$




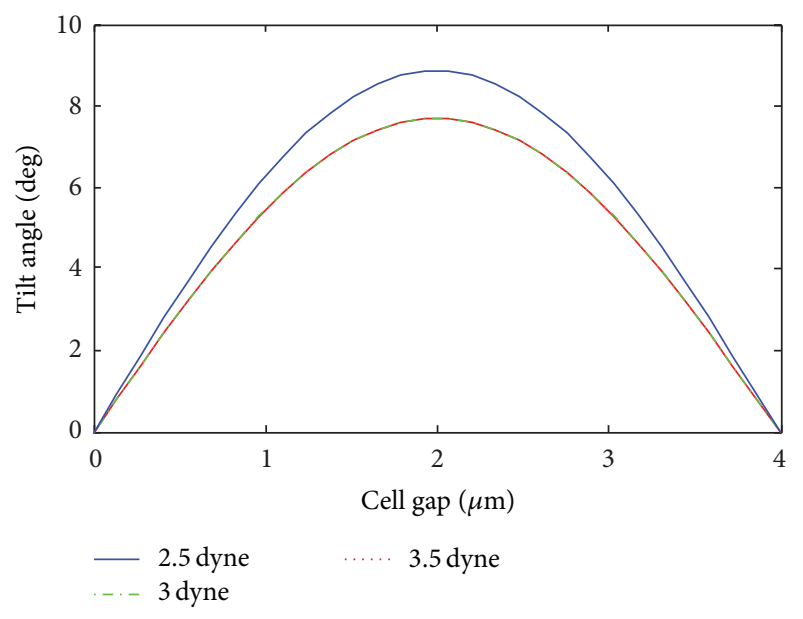

(a)

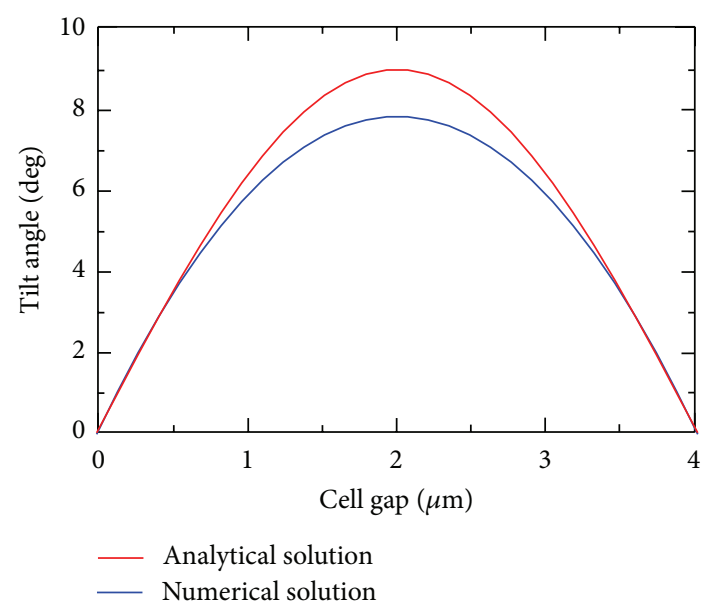

(b)

FIgure 16: (a) Comparison of molecule tilt angles depicted in $4 \mu \mathrm{m}$ cell gap PAA liquid crystals subject to different forces. (b) Comparison of molecule tilt angles subject to 3.5 dyne by analytical and numerical results.

Similarly, we consider the following two cases.

Case $1^{\prime}$

$$
\begin{aligned}
& \left|-\alpha_{2} \frac{\partial v}{\partial y}+\varepsilon_{0} \varepsilon_{a} E^{2} \theta_{m}+F\left(\theta_{m} \cos \phi-\sin \phi\right)\right| \gg\left|K \frac{\partial^{2} \theta_{m}}{\partial x^{2}}\right|, \\
& \gamma \dot{\theta}_{m}=\left(\varepsilon_{0} \varepsilon_{a} E^{2}+F \cos \phi\right) \theta_{m}+\left(-\alpha_{2} \frac{\partial v}{\partial y}-F \sin \phi\right) .
\end{aligned}
$$

Assuming $\dot{\theta}_{m}=a+b \theta_{m}$, one has

$$
\begin{gathered}
a=\frac{1}{\gamma}\left(-\alpha_{2} \frac{\partial v}{\partial y}-F \sin \phi\right), \\
b=\frac{1}{\gamma}\left(\varepsilon_{0} \varepsilon_{a} E^{2}+F \cos \phi\right) .
\end{gathered}
$$

Therefore,

$$
\theta_{m}=\frac{a}{b}\left(e^{b t}-1\right)
$$

is the solution. Substituting (31) into (32) yields

$$
\begin{aligned}
& \theta_{m}(x, t) \\
& =\left[\frac{-\alpha_{2}(\partial v / \partial y)-F \sin \phi}{\varepsilon_{0} \varepsilon_{a} E^{2}+F \cos \phi}\left(e^{\left(\left(\varepsilon_{0} \varepsilon_{a} E^{2}+F \cos \phi\right) / \gamma\right) t}-1\right)\right] \sin \left(\frac{\pi x}{d}\right) .
\end{aligned}
$$

Consider the following.

Case $2^{\prime}$

$$
\left|-\alpha_{2} \frac{\partial v}{\partial y}+\varepsilon_{0} \varepsilon_{a} E^{2} \theta_{m}+F\left(\theta_{m} \cos \phi-\sin \phi\right)\right| \ll\left|K \frac{\partial^{2} \theta_{m}}{\partial x^{2}}\right| .
$$

Since the tactile force and electric field are ignored in Case $2^{\prime}$, only Case $1^{\prime}$ is further examined.

On boundary $y=y_{m}$ in Figure 2,

$$
\begin{aligned}
& \theta_{m}(x, t) \\
& =\left[\frac{\alpha_{2}(\partial v / \partial y)+F \sin \phi}{\varepsilon_{0} \varepsilon_{a} E^{2}+F \cos \phi}\left(e^{\left(\left(\varepsilon_{0} \varepsilon_{a} E^{2}+F \cos \phi\right) / \gamma\right) t}-1\right)\right] \sin \left(\frac{\pi x}{d}\right) .
\end{aligned}
$$

Since $\theta_{m}(x, t)$ in (33) is equal to $-\theta_{m}(x, t)$ in (35), the tilt angles are symmetric.

\section{Discussion}

Subject to both electric field of $8.9 \mathrm{~V}$ and tactile force, simulation results for Case 1 are depicted in Figures 3, 4, and 5. Comparing different LC materials including 5CB, $\mathrm{MBBA}$, and PAA subjected to $10 \mathrm{~ms}$ duration tactile forces of 1 dyne, 0.5 dyne, and 0.1 dyne at $10 \mathrm{~ms}$, Figures 3, 4, and 5 show that a larger force makes the tilt angle larger regardless of LC gap thickness. Figures 6(a), 6(b), and 6(c) compare $3 \mu \mathrm{m}$ thick different LC materials including 5CB, MBBA, and PAA subjected to $5 \mathrm{~ms}$ duration 0.5 dyne force, respectively. The viscosity of PAA is the smallest and the tilt angle is larger than the others. Therefore, the option of liquid crystal material with smaller viscosity can reduce the ripple.

Tilt angle variations are shown under different voltages in Figure 7 and under different tactile forces in Figure 8. Using $10 \mathrm{~V}, 20 \mathrm{~V}, 30 \mathrm{~V}, 40 \mathrm{~V}$, and $50 \mathrm{~V}$ under a tactile force of 3 dyne, it is observed that the tilt angles remain the same in Figures $7(\mathrm{a}), 7(\mathrm{~b})$, and $7(\mathrm{c})$. Thus, different voltages do not affect tilt angles.

Based on (33), Figures 8(a), 8(b), and 8(c) compare different $\mathrm{LC}$ materials including $5 \mathrm{CB}, \mathrm{MBBA}$, and PAA subject to 2.5 dyne, 3 dyne, and 3.5 dyne forces in $3 \mu \mathrm{m}$ and $5 \mu \mathrm{m}$ thick cell gap LC, respectively. Accordingly, tactile forces affect tilt angles obviously. Figures 9(a), 10(a), and 11(a), respectively, show tilt angles of 5CB LC subject to 2.5 dyne, 3 dyne, and 


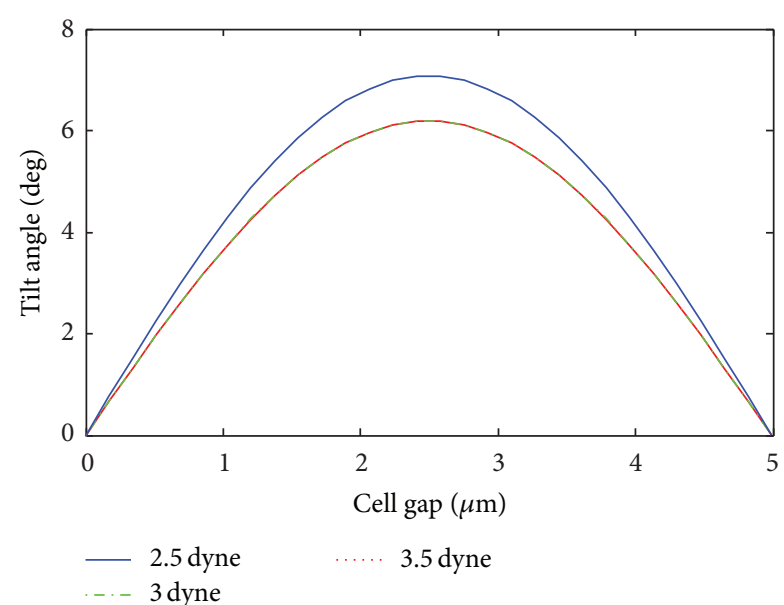

(a)

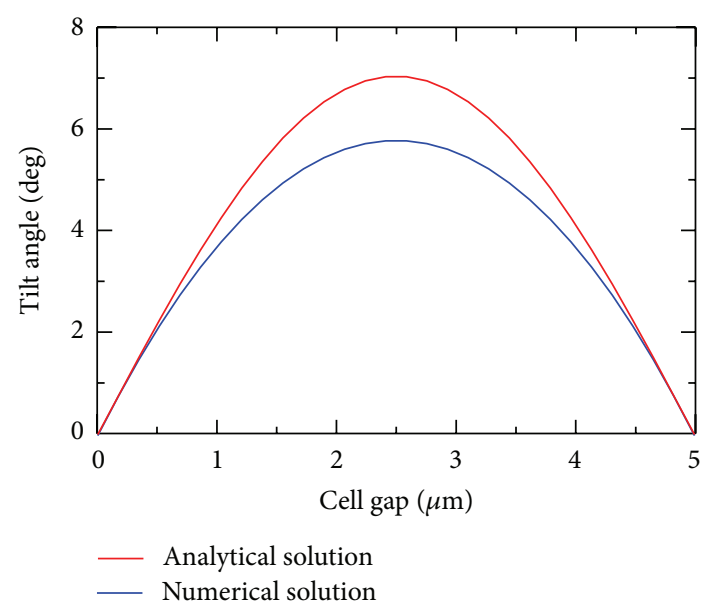

(b)

Figure 17: (a) Comparison of molecule tilt angles depicted in $5 \mu \mathrm{m}$ cell gap PAA liquid crystals subject to different forces. (b) Comparison of molecule tilt angles subject to 3.5 dyne between analytical and numerical results.

3.5 dyne forces in 3,4 , and $5 \mu \mathrm{m}$ thick cell gap LC. Similarly, Figures 12(a), 13(a), and 14(a) are MBBA results and Figures 15(a), 16(a), and 17(a) are PAA results. In addition, Figures 9(b) to 17(b) compare analytical solutions with numerical solutions of the full coupled equations. The comparison results show that analytical solutions are consistent with numerical solutions of the full coupled equations. Therefore, the analytical derivation is validated.

\section{Conclusion}

The Ericksen-Leslie theory deals with the dynamics of nematic liquid crystals. Based on the Ericksen-Leslie theory, this paper contributes to LC dynamics subject to tactile force. LC molecule tilt leads to a change in the optical intensity transmitted through a liquid crystal cell. LCD panels subject to tactile force will show ripple-like propagation on screens. The present results show that the viscosity, tactile force, thickness of cell gap, and Leslie viscosity coefficient are the factors of tilt angle variation. Tilt angle variations of PAA liquid crystal molecules are sensitive to tactile force magnitudes, while those of $5 \mathrm{CB}$ and $\mathrm{MBBA}$ with larger viscosity are not. Analytical derivation has been carried out in this study and analytical results have been validated by numerical results.

\section{Acknowledgments}

This work was supported by Chi Mei Optoelectronics Corp. and Innolux Display Corp. in Taiwan.

\section{References}

[1] J. Ericksen, "Conservation laws for liquid crystals," Transactions of the Society of Rheology, vol. 5, pp. 22-34, 1961.

[2] J. L. Ericksen, "Continuum theory of nematic liquid crystals," Res Mechanica, vol. 21, no. 4, pp. 381-392, 1987.

[3] F. M. Leslie, "Theory of flow phenomenum in liquid crystals," Advances in Liquid Crystals, vol. 4, pp. 1-81, 1979.

[4] F. Brochard, P. Pieranski, and E. Guyon, "Dynamics of the orientation of a nematic-liquid-crystal film in a variable magnetic field," Physical Review Letters, vol. 28, no. 26, pp. 1681-1683, 1972.

[5] L. Lei, S. Changqing, S. Juelian, P. M. Lam, and H. Yun, "Soliton propagation in liquid crystals," Physical Review Letters, vol. 49, no. 18 , pp. 1335-1338, 1982.

[6] F. M. Leslie, "Some topics in continuum theory of nematics," Philosophical Transactions of the Royal Society A, vol. 309, pp. 155-165, 1983.

[7] J. T. Gleeson, P. Palffy-Muhoray, and W. van Saarloos, "Propagation of excitations induced by shear flow in nematic liquid crystals," Physical Review A, vol. 44, no. 4, pp. 2588-2595, 1991.

[8] F. H. Lin and C. Liu, "Existence of solutions for the EricksenLeslie system," Archive for Rational Mechanics and Analysis, vol. 154, no. 2, pp. 135-156, 2000.

[9] L. R. P. de Andrade Lima and A. D. Rey, "Poiseuille flow of Leslie-Ericksen discotic liquid crystal: Solution multiplicity, multistability, and non-Newtonian rheology," Journal of NonNewtonian Fluid Mechanics, vol. 110, no. 2-3, pp. 103-142, 2003.

[10] X. Nie, R. Lu, H. Xianyu, T. X. Wu, and S. T. Wu, "Anchoring energy and cell gap effects on liquid crystal response time," Journal of Applied Physics, vol. 101, no. 10, Article ID 103110, 2007.

[11] P. A. Cruz, M. F. Tomé, I. W. Stewart, and S. McKee, "A numerical method for solving the dynamic three-dimensional Ericksen-Leslie equations for nematic liquid crystals subject to a strong magnetic field," Journal of Non-Newtonian Fluid Mechanics, vol. 165, no. 3-4, pp. 143-157, 2010.

[12] X. Nie, H. Xianyu, R. Lu, T. X. Wu, and S. T. Wu, "Pretilt angle effects on liquid crystal response time," IEEE/OSA Journal of Display Technology, vol. 3, no. 3, pp. 280-283, 2007.

[13] I. C. Khoo and S. T. Wu, Optics and Nonlinear Optics of Liquid Crystals, World Scientific, 1993.

[14] I. C. Khoo, Liquid Crystals: Physical Properties and Nonlinear Optical Phenomena, John Wiley \& Sons, New York, NY, USA, 1995. 


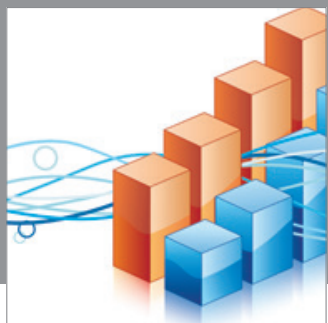

Advances in

Operations Research

mansans

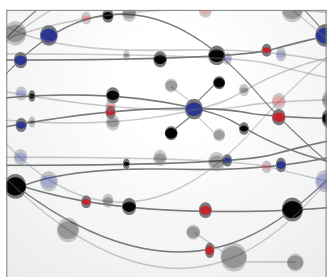

The Scientific World Journal
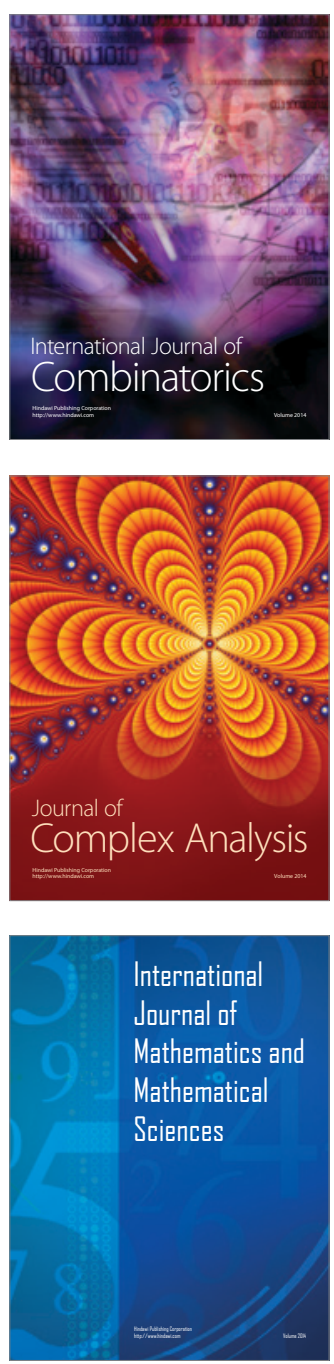
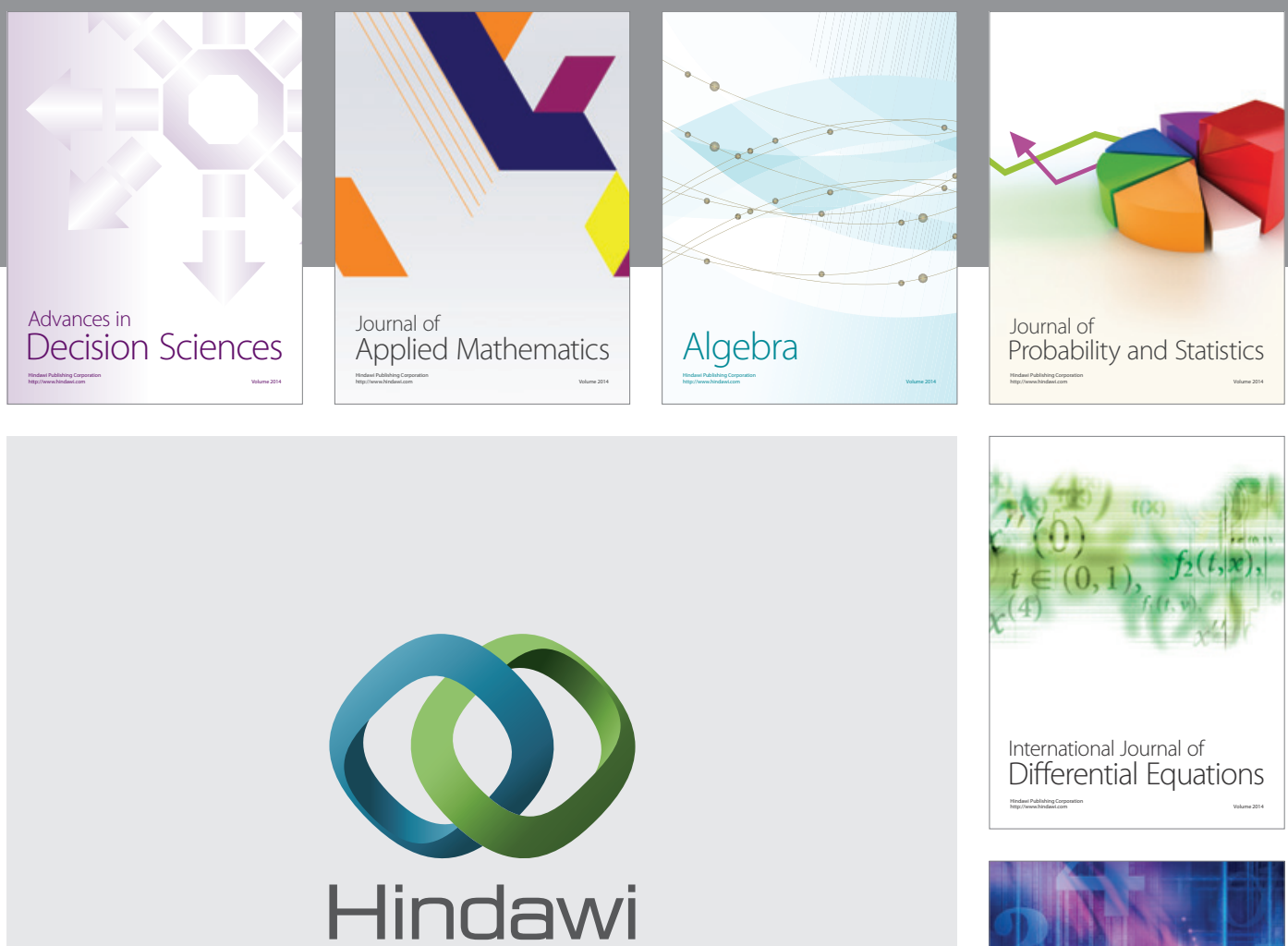

Submit your manuscripts at http://www.hindawi.com
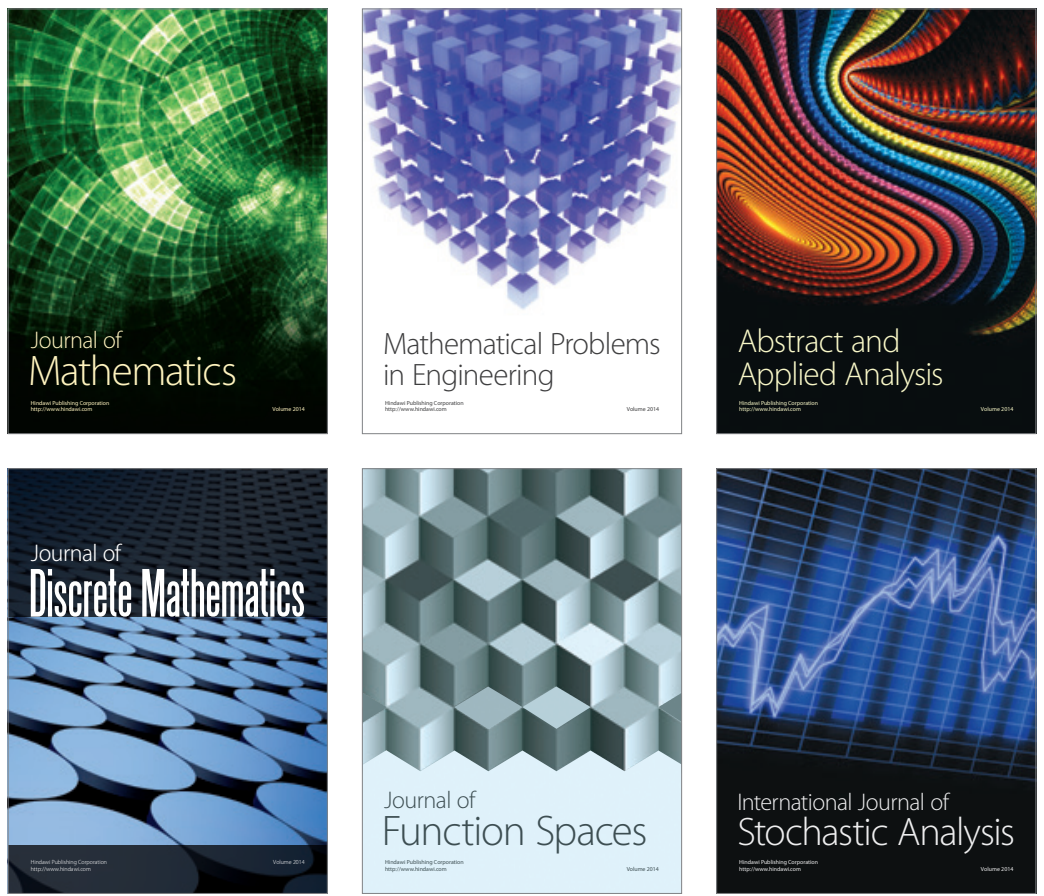

Journal of

Function Spaces

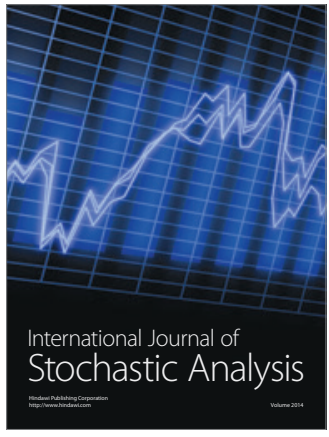

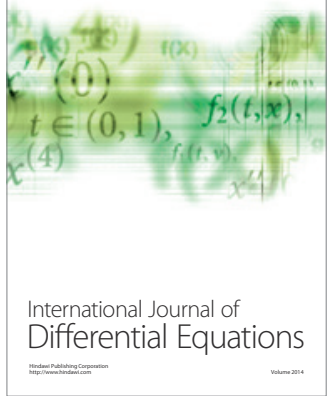
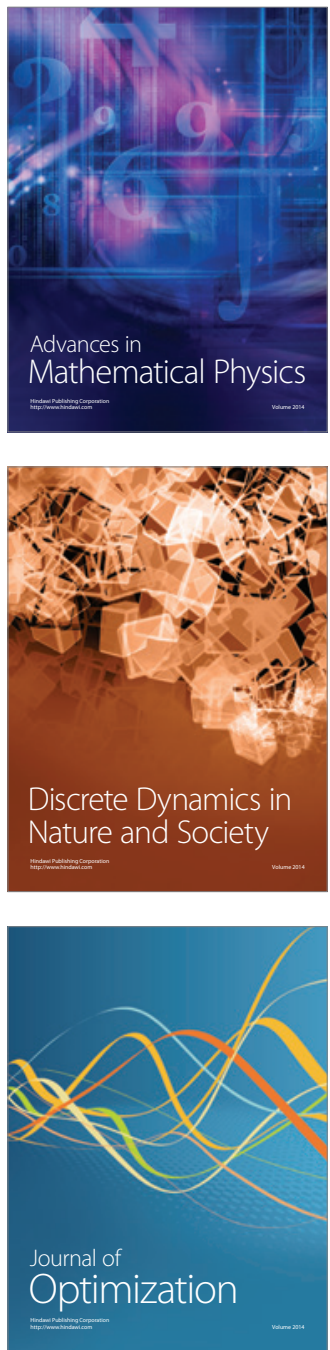